\title{
Digestibility of Dietary Lipids in the Prawn
}

\author{
Shin-ichi Teshima* and Akio KanazawA* \\ (Accepted October 4, 1982)
}

\begin{abstract}
This paper deals with the digestibility of various lipids and cholesterol in the prawn Penaeus japonicus which was determined by an indirect method using chrominum oxide as an indicator. The prawn assimilated the lipids such as pollack liver oil, squid liver oil, soybean oil, oleic acid, palmitic acid, chicken-egg lecithin, tripalmitin, and hydrogenated fish oils effectively; the digestibilities of these lipids were more than $80 \%$ when the diets contained $8 \%$ levels of these lipids. The digestibilities of palmitic acid, tripalmitin, and chicken-egg lecithin were not affected markedly with their levels in the diets. Cholesterol added to the lipid-free diet was absorbed only slightly $(\%$ absorption $=27.5)$. The co-existence of lipids such as palmitic acid, tripalmitin, or chickenegg lecithin markedly improved the absorption rate of cholesterol, indicating the necessity of some lipids for effective assimilation of dietary cholesterol.
\end{abstract}

Crustaceans, unlike vertebrates, are incapable of synthesizing cholesterol and require dietary sources of cholesterol or other sterols for normal growth and survival. ${ }^{1,2}$ Crustaceans also lack the ability for synthesis of bile acids from acetate and cholesterol. ${ }^{3)}$ Interestingly, VAN DEN OORD et $a l^{4,5}$ ) have demonstrated the absence of bile salts and the presence of fatty acyl-sarcosyltaurines in the gastric juice of some crabs as emulsifiers. The above information presumes that the mechanism for assimilation of dietary lipids in crustaceans is different from that found in mammals. ${ }^{6}$ Moreover, recent studies on the nutritional requirements have pointed out that the prawn Penaeus japonicus, the American lobster Homarus americanus, and other crustaceans require essential fatty acids and some phospholipids for growth and survival. ${ }^{2,7)}$ However, little work has been conducted about the digestion and absorption of dietary lipids in crustaceans. ${ }^{8}$. The present study deals with the digestibility of lipids such as triglycerides, fatty acids, phospholipids, and cholesterol in $P$. japonicus and the results are discussed from the nutritional viewpoint.

\section{Materials and Methods}

\section{Feeding of Test Diets and Collection of Feces}

In this study, 3 feeding trials were carried out by using purified diets ${ }^{\theta)}$ containing different types and/or levels of lipids. One group of the prawns
$P$. japonicus was given a lipid-free diet to evaluate the amounts of endogenous lipids excreted into the feces. In experiment 1 , the prawns received the test diets containing $0.5 \%$ cholesterol and $0.8 \%$ level of pollack liver oil, squid liver oil, soybean oil, oleic acid, palmitic acid, chickenegg lecithin (CE-lecithin), or hydrogenated fish oils as lipid sources in order to clarify the digestibility of various lipids. In experiment 2 , the effect of lipid content in diets on the digestibility of lipids and absorption rate of cholesterol was examined with the test diets containing different levels $(4 \%, 8 \%$, and $16 \%$ of palmitic acid, tripalmitin, or CE-lecithin along with $0.5 \%$ cholesterol. In experiment 3 , the effects of CE-lecithin on the digestibility of tripalmitin and absorption rate of cholesterol were investigated.

The prawns, $20-30 \mathrm{~g}$ in each body weight, were divided into lots of 5 prawns in a tank $(30 \mathrm{l})$ and fed the test diets at the 3.0\% level of their body weight daily. ${ }^{10}$ The 6-day pools of feces were dried for analysis of lipid and cholesterol contents. Every test diet contained the same ingredients $\left.(\mathrm{g} / 100 \mathrm{~g} \mathrm{diet})^{9}\right)$ except for lipid sources: glucose 5.5 , sucrose 10.0 , $\alpha$-starch 4.0 , glucosamine $\mathrm{HCl}$ 0.8 , casein 50.0 , sodium citrate 0.3 , sodium succinate 0.3 , minerals 8.5 , vitamins 3.2 , agar 3.0 , and chromic oxide (as an indicator) 0.5 . The total proportion of diets was adjusted to $100 \%$ with cellulose powder.

* Faculty of Fisheries, University of Kagoshima, 50-20 Shimoarata-4, Kagoshima 890, Japan（手島新一 • 金沢昭夫: 鹿兔島大学水産学部). 
Measurements of Digestibility of Lipids and Absorption Rate of Cholesterol

The digestibility of saponifiable lipids was determined by an indirect method using chromic oxide as an indicator. Apparent digestibility $(\%)$ was given by the equation (1). ${ }^{11}$ True digestibility $(\%)$ can be obtained by the equation (2) by correcting the equation (1) for endogenous lipids.

Apparent digestibility

$$
(\%)=\left[1-\left(\frac{a^{\prime}}{a} \times \frac{b}{b^{\prime}}\right)\right] \times 100
$$

True digestibility

$$
\left.(\%)=\left[1-\left(\frac{a^{\prime}}{a} \times \frac{b}{b^{\prime}}\right)+\frac{b}{b_{0}{ }^{\prime}} \times \frac{e}{a}\right)\right] \times 100
$$

a: lipid $(\%)$ of dry diet

$a^{\prime}: \quad$ lipid $(\%)$ of dry feces

$b$ : chromic oxide (\%) of dry diet

$b^{\prime}: \quad$ chromic oxide $(\%)$ of dry feces

$b_{0}{ }^{\prime}:$ chromic oxide $(\%)$ of dry feces when the prawns received a lipid-free diet

$e: \quad$ lipid $(\%)$ of dry feces when the prawns received a lipid-free diet

Apparent and true adsorption rates of cholesterol were also determined by a similar manner according to the equations (1) and (2).

The feces were refluxed with chloroformmethanol (2:1) twice to give lipids. The residue was subjected to determination of chromic oxide content by the acid-digestion method of FURUKAWA and TSUKAHARA. ${ }^{12}$ Lipid and cholesterol contents were determined by BRAGDON's method ${ }^{13)}$ and by SPERRY and WeBB's method, ${ }^{14}$ ) respectively. Triglyceride and phospholipid contents were measured by AMENTA's method ${ }^{152}$ after separation of lipids into lipid classes by thinlayer chromatography on Silica gel G. ${ }^{16)}$

\section{Results and Discussion}

In experiment 1 , the digestibility of various lipids was examined. Table 1 shows the apparent and true digestibilities of lipids in the prawns which were fed the diets containing $8.0 \%$ level of respective lipids. The apparent digestibilities of lipids were lower by about $6 \%$ than the true digestibilities. Although oleic acid, pollack liver oil, and hydrogenated fish oils seemed to be assimilated slightly less than squid liver oil, palmitic acid, and CE-lecithin, the true digesti-
Table 1. Digestibility of various types of lipids in

\begin{tabular}{|c|c|c|}
\hline \multirow{2}{*}{ Lipid*1 } & \multicolumn{2}{|c|}{$\begin{array}{c}\text { Digestibility } \\
(\%)\end{array}$} \\
\hline & Apparent & True \\
\hline Pollack liver oil & 78.4 & 83.9 \\
\hline Squid liver liver oil & 83.9 & 89.4 \\
\hline Soybean oil & 81.1 & 86.6 \\
\hline Oleic acid & 77.8 & 83.3 \\
\hline Palmitic acid & 87.9 & 93.4 \\
\hline Chicken-egg lecithin & 88.0 & 91.5 \\
\hline $\begin{array}{l}\text { Hydrogenated fish oil A } \\
\left.\text { (m.p. } 36.0^{\circ} \mathrm{C}\right)^{* 2}\end{array}$ & 75.2 & 80.7 \\
\hline $\begin{array}{l}\text { Hydrogenated fish oil B } \\
\left.\text { (m.p. } 44.6^{\circ} \mathrm{C}\right)^{* 2}\end{array}$ & 77.7 & 83.2 \\
\hline
\end{tabular}
P. japonicus

*1 To determine the digestibiliry of lipids, the prawns were fed the test diets containing $0.5 \%$ cholesterol and $8.0 \%$ level of one of the saponifiable lipids as lipid sources. In addition, 2 groups of the prawns were fed the lipid-free and $0.5 \%$ cholesterol-containing diers, respectively, to evaluate the amounts of endogenous saponifiable lipids and cholesterol.

*2 Hydrogenated fish oils A (IV $=80.4)$ and B (IV $=51.5)$ were kindly supplied from Riken Vitamin Co.

bilities of lipids examined were more than $80 \%$. This indicates that the prawn $P$. japonicus is capable of assimilating every lipid examined effectively. As for the rainbow trout, TAKEUCHI et al. $^{17)}$ have shown that the digestible energy of diets decreased by the inclusion of a hydrogenated fish oil, but such a decrease in digestible energy was small when the hydrogenated fish oil was used with cuttlefish liver oil as dietary lipid sources. In the case of the prawn, interestingly, the hydrogenated fish oils $\mathbf{A}$ and $\mathbf{B}$ gave the high digestibilities. This suspects that $P$. japonicus may be able to utilize hydrogenated fish oils as a dietary energy source when the lipids containing sufficient amounts of essential fatty acids were supplemented to diets, as pointed out with the rainbow trout and carp by TAKEUCHI et al. ${ }^{13}$ )

The results of experiment 2 are shown in Tables 2 and 3 . The digestibilities of palmitic acid, tripalmitin, and CE-lecithin did not vary markedly with dietary levels, however the digestibility of triplamitin was slightly decreased when $16 \%$ tripalmitin was added to the diet (Table 2). ANDREws and Sick ${ }^{18}$ ) have suggested that lipase activity in crustaceans is possibly low because Penaeus setiferus incorporated palmitic acid more efficiently than tripalmitin into the body. Whereas, the prawn $P$. japonicus is suggested to have a high lipase activity, because the digestibilities of tripalmitin, pollack liver oil, squid liver oil, and 
Table 2. Effect of dietary lipid levels on the digestibility of palmitic acid, tripalmitin, and chickenegg lecithin

\begin{tabular}{lccc}
\hline \multirow{2}{*}{ Dietary lipid } & \multicolumn{3}{c}{ True digestibility (\%) } \\
\cline { 2 - 4 } & \multicolumn{3}{c}{ Lipid level (\%) } \\
\cline { 2 - 4 } & 4.0 & 8.0 & 16.0 \\
\hline Palmitic acid & 89.6 & 95.1 & 93.2 \\
Tripalmitin & 90.1 & 89.2 & 82.0 \\
Chicken-egg lecithin & 98.8 & 96.3 & 94.3 \\
\hline * The diets contained $0.5 \%$ cholesterol in addition to the \\
respective lipids as lipid sources.
\end{tabular}

Table 3. Effect of saponifiable lipids on the absorption of cholesterol

\begin{tabular}{lcccc}
\hline \multirow{2}{*}{ Dietary lipid } & \multicolumn{3}{c}{$\begin{array}{c}\text { True absorption (\%) of } \\
\text { cholesterol }\end{array}$} \\
\cline { 2 - 5 } & \multicolumn{3}{c}{ Lipid level (\%) in diets* } \\
\cline { 2 - 5 } & 0 & 4.0 & 8.0 & 16.0 \\
\hline Palmitic acid & 27.5 & 94.6 & 98.2 & 91.0 \\
Tripalmitin & 27.5 & 85.8 & 94.1 & 84.7 \\
Chicken-egg lecithin & 27.5 & 90.9 & 92.9 & 93.4 \\
\hline * The diets contained & $0.5 \%$ cholesterol in addition to the \\
respective lipids.
\end{tabular}

soybean oil were not low notably as compared with those of free fatty acids (Tables 1 and 2).

Table 3 shows the effect of dietary lipids on the absorption of cholesterol. When the diet contained no lipid except cholesterol, the absorption rate of cholesterol was only $27.5 \%$. The absorption of cholesterol was improved markedly by the addition of palmitic acid, tripalmitin, or CE-lecithin, and more than $80 \%$ cholesterol was absorbed when the diets contained these lipids at livel of $4 \%, 8 \%$, or $16 \%$. Thus, the present study shows that the inclusion of lipids is necessary for efficient absorption of dietary cholesterol from the alimentary tracts in P. japonicus.

In mammals, micellar solubilization is an essential step for efficient assimilation of dietary lipids. Deshimaru ${ }^{18)}$ has observed that growth of the prawn $P$. japonicus was improved by the addition of ursodeoxycholic acid $(3 \alpha, 7 \beta$-dihydroxy- $5 \beta$-cholonoic acid) to diets. LeSTER et $a l .^{20)}$ have examined the single-phase micellar formation by the ternary system of cholesterol, lecithin, and $\mathrm{N}$-(N-dodecanoylarcosyl) taurine (DST: a representative emulsifier in the gastric juice of crabs). They have shown that cholesterol solubilization was increased by the addition of lecithin to DST. Recent nutritional studies have demonstrated that the addition of phospholipids to diets was effective in improving growth and survival of $P$. japonicus ${ }^{7,18,21)}$ and the American lobster Homarus americanus. ${ }^{22)}$ These findings suspect that phospholipids are likely to play an important role in the assimilation of dietary lipids and cholesterol in crustaceans. In experiment 3 , therefore, the effects of CE-lecithin on the digestibility of tripalmitin and absorption of cholesterol were investigated. In addition, since the presence of glucosamine $\mathrm{HCl}$ in diets has been reported to reduced the growth-promoting effect of cholesterol in P. japonicus, ${ }^{23)}$ the effect of glucosamine $\mathrm{HCl}$ on the absorption of cholesterol was examined.

The results of experiment 3 are given in Table 4. Cholesterol was not absorbed effectively from the alimentary tracts when the diet contained neither tripalmitin nor CE-lecithin as observed in experiment 2. The addition of $2.0 \%, 4.0 \%$, or $8.0 \%$ CE-lecithin to diets enhanced the absorption of cholesterol markedly. However, not only CE-lecithin but also tripalmitin enhanced the absorption of cholesterol: the high absorp-

Table 4. Effects of chicken-egg lecithin and glucosamine $\mathrm{HCl}$ on the true digestibility of tripalmitin and absorption of cholesterol

\begin{tabular}{|c|c|c|c|c|c|}
\hline \multicolumn{4}{|c|}{ Lipid and glucosamine levels $(\%)$ in diets } & \multicolumn{2}{|c|}{ Digestibility or absorption (\%) } \\
\hline $\begin{array}{c}\text { Chicken-egg } \\
\text { lecithin }\end{array}$ & Tripalmitin & Cholesterol & $\begin{array}{c}\text { Glucosamine } \\
\mathrm{HCl}\end{array}$ & Tripalmitin & Cholesterol \\
\hline 0 & 0 & 0.5 & 0 & - & 30.0 \\
\hline 4.0 & 0 & 0.5 & 0 & - & 98.6 \\
\hline 8.0 & 0 & 0.5 & 0 & - & 91.3 \\
\hline 8.0 & 0 & 0.5 & 0.8 & - & 90.0 \\
\hline 0 & 8.0 & 0.5 & 0 & - & 93.3 \\
\hline 0.5 & 8.0 & 0.5 & 0 & 90.2 & 93.0 \\
\hline 1.0 & 8.0 & 0.5 & 0 & 99.0 & 92.0 \\
\hline 2.0 & 8.0 & 0.5 & 0 & 98.0 & 91.5 \\
\hline 4.0 & 8.0 & 0.5 & 0 & 99.7 & 95.0 \\
\hline
\end{tabular}


tion rate of cholesterol was also observed on the diets containing $8.0 \%$ tripalmitin alone, or both $8.0 \%$ tripalmitin and varying levels of $\mathrm{CE}$-lecithin as coexistent lipids. Also, the absorption rate of cholesterol did not vary notably with the levels of CE-lecithin and/or tripalmitin in diets. On the other hand, the digestibility of tripalmitin was affected by the levels of CE-lecithin in diets. The digestibility of tripalmitin was increased with the increasing levels of CE-lecithin in diets. This assumes that a high dietary value of phospholipids for the prawn ${ }^{212}$ and American lobster ${ }^{222}$ is due partially to their contribution in improving the assimilation of dietary lipids such as triglycerides but not cholesterol. However, we assume that dietary phospholipids exert a high dietary value for the crustaceans due mainly to some other mechanism: for example, dietary phospholipids are necessary for the harmonious transport of dietary lipids and cholesterol through the hemolymph, because high density lipoproteins rich in phospholipids have been shown to play an important role in the transport of dietary lipids in the prawn $P$. japonicus. ${ }^{8}$ )

The supplement of glucosamine $\mathrm{HCl}$ had no effect on the absorption rate of cholesterol. Therefore, the question remains about the mechanism by which glucosamine $\mathrm{HCl}$ reduces the growth-promoting effect of cholesterol in $P$. japonicus. ${ }^{23)}$

\section{References}

1) A. Kanazawa, N. Tanaka, S. Teshima, and K. Kashiwada: Bull. Japan. Soc. Sci. Fish., 37, 211-215 (1971).

2) S. Teshima: in "Yogyo to Shiryo-Shishitsu" (ed. by Japan. Soc. Sci. Fish.), Suisangaku Series, No. 22, Koseisha Koseikaku, Tokyo, 1978, pp. 60-77.

3) D. A. Holwerda and H.J. Vonk: Comp. Biochem. Physiol., 45B, 51-58 (1973).

4) A. VAN DEN OORd, H. Danielson, and R. RYHAGE: Nature, 203, 301 (1964).
5) A. Van den Oord, H. Danielson, and $R$. RyHAGe: J. Biol. Chem., 240, 2242-2247 (1965).

6) R. B. Goldrick: in "Biochemistry and Methodology of Lipids" (ed. by A. R. JoHNSON and J. B. DAVENPORT), Wiley-Interscience, New York, London, Sydney, and Toronto, 1971, pp. 501-514.

7) A. Kanazawa: Proceeding of 2nd Int. Conf. on Aquaculture Nutrition, in press.

8) S. Teshima and A. Kanazawa: Mini Rev. Data File of Fish. Res., Kagoshima Univ., 1, 1-25 (1980).

9) A. Kanazawa, S. Teshima, and S. Tokrwa: Bull. Japan. Soc. Sci. Fish., 43, 849-856 (1977).

10) S. Teshima, A. Kanazawa, and H. OKamoto: Bull. Japan. Soc. Sci. Fish., 40, 1015-1019 (1974).

11) L. A. MAYNARD and J. K. Loostr: "Animal Nutrition", 6th ed., McGraw-Hill Book Co., New York, 1969, pp. 352-353.

12) A. Furukawa and H. Tsukahara: Bull. Japan. Soc. Sci. Fish., 32, 502-506 (1966).

13) J. H. BRAGDON: in "Lipids and Steroid Hormones in Clinical Medicine" (ed. by F. W. Sunderman), Lipincott, Philadelphia and Montreal, 1960, pp. 9-14.

14) W. M. SPERRY and M. WebB: J. Biol. Chem., 187, 97-106 (1950).

15) J. S. AMENTA: J. Lipid Res., 5, 270-272 (1964).

16) V. P. SkIPSI, A. F. SMolowe, R. C. Sulivan, and M. BARClay: Biochim. Biophys. Acta, 106, 386196 (1965).

17) T. Takeuch, T. Watanabe, and C. Ogino: Bull. Japan. Soc. Sci. Fish., 44, 875-881 (1978).

18) J.W. Andrews and L. V. Sick: Proc. World Maricult. 3rd Annual Workshop., 403-414 (1972).

19) O. Deshimaru: Mem. Kagoshima Pref. Fish. Exptl. Station, 12, 1-118 (1981).

20) R. Lester, M. C. CAREY, J. M. LitTLE, and S. R. Dowd: Science, 189, 1098-1100 (1975).

21) A. Kanazawa, S. Teshima S. Tokiwa, M. Endo, and F. A. ABDEL RAZEK: Bull. Japan. Soc. Sci. Fish., 45, 961-965 (1979).

22) L. R. D'Abramo, C. E. Bordner, D. E. Conkrin, and N. A. BAUM: J. Nutrition, 111, 425-431 (1981).

23) O. Deshimaru and K. Kuroki: Bull. Japan. Soc. Sci. Fish., 40, 421-424 (1974). 